\title{
Expressed transcripts associated with high rates of egg production in chicken ovarian follicles
}

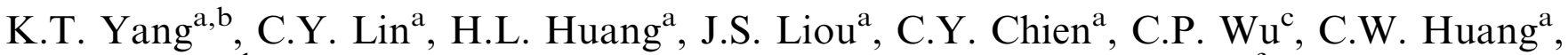 \\ B.R. Ou ${ }^{d}$, C.F. Chen ${ }^{\mathrm{a}}$, Y.P. Lee ${ }^{\mathrm{a}}$, E.C. Lin ${ }^{\mathrm{e}}$, P.C. Tang ${ }^{\mathrm{a}}$, W.C. Lee ${ }^{\mathrm{f}}$, S.T. Ding ${ }^{\mathrm{e}}$, \\ W.T.K. Cheng ${ }^{\mathrm{e}}$, M.C. Huang ${ }^{\mathrm{a}, *}$ \\ ${ }^{a}$ Department of Animal Science, National Chung Hsing University, 250 Kuo-Kuang Road, Taichung, Taiwan \\ b Institute of Biomedical Sciences, Academia Sinica, 182 Section 2, Academia Road, Nankang, Taipei, Taiwan \\ ${ }^{\mathrm{c}}$ Department of Animal Science, National Chiayi University, 300 Syuefu Road, Chiayi, Taiwan \\ ${ }^{\mathrm{d}}$ Department of Animal Science and Biotechnology, Tunghai University, 181 Taichung Harbor Road, Section 3, Taichung, Taiwan \\ ${ }^{\mathrm{e}}$ Department of Animal Science and Technology, National Taiwan University, 50, Lane 155, Kee-Lung Road, Taipei, Taiwan \\ ${ }_{\mathrm{f}}^{\mathrm{f}}$ Animal Technology Institute Taiwan, 52, Kedung 2nd Road, Ding-Pu Lii, Chunan, Miaoli, Taiwan
}

Received 19 June 2006; accepted 12 June 2007

Available online 27 June 2007

\begin{abstract}
The purpose of this study was to characterize differentially expressed transcripts associated with varying rates of egg production in Taiwan country chickens. Ovarian follicles were isolated from two strains of chicken which showed low (B) or high (L2) rates of egg production, then processed for RNA extraction and cDNA library construction. Three thousand and eight forty clones were randomly selected from the cDNA library and amplified by PCR, then used in microarray analysis. Differentially expressed transcripts $(P<0.05$, $\left.\log _{2} \geqslant 1.75\right)$ were sequenced, and aligned using GenBank. This analysis revealed 20 non-redundant sequences which corresponded to known transcripts. Eight transcripts were expressed at a higher level in ovarian tissue prepared from chicken strain B, and 12 transcripts were expressed at a higher level in L2 birds. These differential patterns of expression were confirmed by semi-quantitative RT-PCR. We show that transcripts of cyclin B2 (cycB2), ferritin heavy polypeptide 1 (FTH1), Gag-Pol polyprotein, thymosin $\beta 4$ (TB4) and elongation factor $1 \alpha 1$ (EEF1A1) were enriched in B strain ovarian follicles. In contrast, thioredoxin (TXN), acetyl-CoA dehydrogenase long chain (ACADL), inhibitor of growth family member 4 (ING4) and annexin II (ANXA2) were expressed in at higher levels in the L2 strain. We suggest that our approach may lead to the isolation of effective molecular markers that can be used in selection programs in Taiwan country chickens.
\end{abstract}

(C) 2007 Elsevier Ltd. All rights reserved.

Keywords: Chicken; Ovarian follicles; Egg production; cDNA microarray; RT-PCR

\section{Introduction}

Traditional breeding strategies designed to improve the efficiency of chicken egg production are based on long term monitoring of egg number and laying rate; however, such approaches are extremely time consuming [1]. We have studied two strains of domestic Taiwan country chicken which have been established over 20 generations of

\footnotetext{
*Corresponding author. Tel.: + $886422840366 \times 239$; fax: +886422860265

E-mail address: mchuang@mail.nchu.edu.tw (M.C. Huang).
}

selection from a common base population [2]. These two strains of chicken have significantly different rates of egglaying. The L2 strain was mainly selected for egg production, whilst the $\mathrm{B}$ strain was selected for meat production. We took advantage of this selection effect and defined the B and L2 chicken strains to be low or high rate egg production groups, respectively.

The normal physiological phenomenon of egg production takes place because of complex sets of interactions between genes regulating energy metabolism, protein synthesis, and storage processes, and is dependent on participation in this process by many different organs. 
The ovary is particularly important in this process as it produces various hormones that regulate oocyte and follicle maturation and ovulation $[3,4]$. Previous studies have identified several factors required for chicken egg production $[5,6]$, however, the composition of the chicken ovarian transcriptome has not yet been fully defined, and the major regulatory genes or interactions governing oogenesis and follicle development remain to be identified.

As existing breeding programs take a long time, it is an important goal to develop a rapid and accurate selection method. In the mid-1990s, cDNA microarray technology was developed and permitted the large scale analysis of patterns of gene expression [7,8]. However, most microarray studies have been carried out in humans and rodents rather than in livestock animals [9,10]. Furthermore, although studies of gene expression in oocytes and follicles have been published in mammals such as cows [11,12] and pigs $[13,14]$ there is little data related to poultry. Analysis of expressed sequence tags (ESTs) from tissue-specific cDNA libraries has provided a rapid and efficient approach for gene discovery [15]. Some chicken ESTs have been identified in this way and have been obtained from libraries derived from spleen $T$ cells [16], the Bursa of Fabricius [17], 10 day embryos [18,19] and the liver [20]. Thus cDNA microarrays have been successfully used to profile gene expression patterns in different organs [21,22], but have yet to be used to study strain specific differences that may be associated with particular traits, such as rates of egg-laying.

In this study, transcriptional profiling with cDNA microarrays was used to identify differences in gene expression between ovaries from two strains of Taiwan country chickens. We have identified candidate molecular markers that correlate with low or high rates of egg production and postulate that these differentially expressed genes have the potential to be used as tools in future breeding strategies.

\section{Materials and methods}

\subsection{Tissue collection and RNA preparation}

Thirty chickens (15 of each strain) were sacrificed at 56weeks of age, and the ovarian tissue, which contained various sizes of egg follicles, was collected post egg-laying. The egg yolk of the follicles was removed before storing the rest of the ovarian tissue in RNAlater (Ambion ${ }^{\circledR}$, Austin, Texas, USA) at $-80^{\circ} \mathrm{C}$. Total RNA was isolated from this tissue using the RareRNA reagent (GenePure, Kaysville, UT, USA) in accordance with the manufacturer's instructions. Equal amounts of total RNA extracted from three samples of each chicken strain were pooled together, then mRNA was purified from this shared material using the mRNA purification kit (Ambion ${ }^{\circledR}$, Austin, Texas, USA). This pooled mRNA was used for cDNA library construction. The total RNA isolated from the 12 other individuals of each strain were used for microarray hybridization analysis or semi-quantitative RT-PCR analysis.

\subsection{Chicken ovarian follicle cDNA library construction}

cDNA libraries were constructed from ovarian follicles of both strains of chicken using the SuperScript ${ }^{\mathrm{TM}}$ Plasmid System and Gateway ${ }^{\circledR}$ Technology for cDNA Synthesis and Cloning (Invitrogen ${ }^{\mathrm{TM}}$, Carlsbad, CA, USA). Five micrograms of mRNA purified from the pooled follicular tissue were reverse transcribed to generate template cDNA to use in the library construction. The cDNA transcripts were size-fractionated, then directionally cloned into the SalI and NotI sites of a pre-digested plasmid vector, pCMV.SPORT6.1 (Invitrogen ${ }^{\mathrm{TM}}$, Carlsbad, CA, USA) and electroporated into E. coli DH10B competent cells (Invitrogen $^{\mathrm{TM}}$, Carlsbad, CA, USA) (Gene Pulser ${ }^{\mathrm{TM}}$, BioRad, Hercules, CA, USA). The transformed cDNA library was titrated then mixed with sterilized glycerol for long term storage at $-70{ }^{\circ} \mathrm{C}$.

\section{3. cDNA microarray assembly}

Randomly selected 3840 colonies were picked from the plated cDNA libraries and cultured in LB medium supplemented with $50 \mu \mathrm{g} / \mathrm{ml}$ ampicillin (Athena Enzyme System $^{\text {TM }}$, USA) at $37^{\circ} \mathrm{C}$ for $16-18 \mathrm{~h}$. Plasmid DNA was isolated from overnight cultures using the Montage ${ }^{\mathrm{TM}}$ Plasmid Miniprep $_{96}$ Kit (Millipore Corporation, Billerica, MA, USA). The cDNA inserts present in recombinant plasmids were amplified by PCR in 96-well microplates and a GeneAmp 2700 machine (Applied Biosystems, Foster City, CA, USA). The inserts were amplified using the SP6 (5'-ATT TAG GTG ACA CTA TAG-3') and T7 (5'-TAA TAC GAC TCA CTA TAG GG-3') primers which flanked the cloning site. PCR products were purified by isopropanol precipitation, washed with $70 \%$ ethanol, resuspended in $20 \mu \mathrm{l}$ of $50 \%$ dimethyl sulfoxide (DMSO) then transferred to 384-well microplates as microarray sources for printing. All the 3840 clones were printed onto CMTGAPS II glass slides (Corning, NY, USA) using a OmniGrid Accent ${ }^{\mathrm{TM}}$ microarrayer (GeneMachine, Bethesda, MD, USA) equipped with 8 spotting pins (ArrayIt ${ }^{\circledR}$, TeleChem, International, Inc., Sunnyvale, CA, USA). The microarrays were designed so that each amplicon was spotted twice so there were 7680 spots in each slide (196 spots in each subarray). The printed slides were UV crosslinked at $1200 \mathrm{~mJ} / \mathrm{cm}^{2}$ of energy (Stratagene Stratalinker) and stored at room temperature in a dry incubator.

\subsection{Target labeling and hybridization}

Forty micrograms of total RNA isolated from ovarian follicles taken from either the L2 $(n=6)$ or B $(n=6)$ chicken strains were used as the template for a reverse transcription reaction which incorporated the fluorescent nucleotide Cy3-dUTP into the cDNA product using the 
MicroMax Direct Labeling Kit (PerkinElmer, Inc., Wellesley, MA, USA). This fluorescent cDNA product was purified by removing unincorporated fluorescent marker with isopropanol precipitation and washing in $70 \%$ ethanol. The purified cDNA pellet from each strain was dissolved in $20 \mu \mathrm{l}$ of Hybridization Buffer (PerkinElmer, Inc., Wellesley, MA, USA) at room temperature for $10 \mathrm{~min}$, before denaturing at $90^{\circ} \mathrm{C}$ for $2 \mathrm{~min}$ immediately before hybridization. The denatured labeled cDNA targets were pipetted onto the microarrays which were covered with glass cover slips $(24 \times 40 \mathrm{~mm}$, Matsunami Glass Ind Ltd, Tokyo, Japan) to prevent evaporation. Hybridization was performed overnight in a sealed hybridization chamber (GeneMachine, Bethesda, MD, USA) at $65^{\circ} \mathrm{C}$, under humidified conditions. Following hybridization, the slides were washed sequentially in $0.5 \mathrm{X}$ SSC $/ 0.1 \%$ SDS for $15 \mathrm{~min}, 0.06 \mathrm{X} \mathrm{SSC} / 0.1 \% \mathrm{SDS}$ for $15 \mathrm{~min}$ and $0.06 \mathrm{X} \mathrm{SSC}$ for $15 \mathrm{~min}$, all at room temperature with gentle agitation. The washed slides were dried by placing them individually in $50 \mathrm{ml}$ centrifuge tubes, then spinning for $2 \mathrm{~min}$ at $2000 \mathrm{rpm}$.

\section{5. cDNA microarray data analysis}

After being washed and dried the microarray slides were scanned immediately using a GenePix 4000B scanner (Axon, Instruments Inc., Foster City, CA, USA) at $532 \mathrm{~nm}$ to detect the fluorophore Cy3. The GenePix Pro 4.1 version (Axon, Instruments Inc., Foster City, CA, USA) was utilized to acquire the array image and then quantify the microarray data. Each microarray spot was defined by its position within a grid of circles placed over the array image, and the whole image was processed to record the pattern of spot intensities across the slide. This raw data was imported into the commercial Avadis analysis software (version 3.3; Strand Life Sciences, Redwood City, CA, USA). Poor quality median intensity data $($ flag $\neq 0)$ was removed before analysis, and then the remaining information was subjected to background subtraction and Quantile global normalization. Net intensities less than 500 were excluded and the residual signal were $\log$ transformed for further differential statistical expression analysis using the unpaired $t$-test with a p-value cutoff value of 0.05 . Transcripts were considered to be differentially expressed when the mean value of $\log _{2}$ ratio was 1.75 -fold greater, or lesser than -1.75 -fold, thus representing a greater than 3-fold change in expression between B or L2 ovarian follicles.

\subsection{Sequencing and data processing}

Sequencing reactions were performed using the BigDye Terminator Cycle Sequencing Kit (version 3.1, Applied Biosystems, Foster City, CA, USA) with the SP6 forward primer (5'-ATT TAG GTG ACA CTA TAG-3'). Sequences were analyzed on an ABI 3100 genetic analyzer (Applied Biosystems, Foster City, CA, USA). Sequence data were uploaded to the Phred cross match program, using the Phrap software package for quality assessment and vector sequence trimming (http://www.phrap.org/) [23]. Cluster analysis was performed using the TGI Clustering tool software to identify unique sequences (http://www.tigr.org/tdb/software). Finally, sequences were aligned with the basic local alignment search tool (BLAST), and screened against the NCBI nucleotide database using the BLASTN program (http://www.ncbi. nlm.gov/BLAST/).

\subsection{Semi-quantitative $R T-P C R$ analysis}

Semi-quantitative RT-PCR was performed to validate the differentially expressed transcripts identified from the cDNA microarray following the method described in [24]. Total RNAs isolated as described above were reverse transcribed to generate first strand cDNA using the MasterAmp $^{\text {TM }}$ High Fidelity RT-PCR Kit (Epicentre ${ }^{\circledR}$ Biotechnologies, Madison, WI, USA). The PCR primers used are listed in Table 1 and were designed using Primer Express version 2.0 software (Applied Biosystems, Foster City, CA, USA). PCR was carried out for $25,30,35$ or 40 cycles when the cycle number was proportional to the DNA yield. The PCR products were separated by electrophoresis on a $3 \%$ agarose gel, and visualized by staining with ethidium bromide. The $18 \mathrm{~S}$ rRNA product acted as the positive control during amplification.

\section{Results}

\section{1. cDNA microarray construction and data analysis}

cDNA libraries were constructed from both the L2 and B strains of chicken. Each library contained $4 \times 10^{5}$ independent clones, and in total 3840 clones were randomly selected from the cDNA libraries for further analysis. The average size of cloned inserts in the library ranged from 0.5 to $1.5 \mathrm{~kb}$ (Fig. 1). The amplicons from the 3840 anonymous clones were spotted out in duplicate onto glass slides and so the resultant cDNA microarrays consisted of 7680 elements. Slide-to-slide reproducibility and efficiency of the nucleic hybridization protocol was assessed using a quality control test in which two identical samples were labeled directly with Cy3-dUTP then the labeled material distributed equally between each slide. If our method was effective there should be no significant difference between the hybridization patterns observed on the two slides, and this was indeed the case.

Twelve chicken ovarian follicle cDNA microarrays were used (six arrays of each chicken strain) in our experiments to identify differentially expressed transcripts in ovarian follicles from the chicken strains associated with either low (B) or high (L2) rates of egg production. Most hybridization spots had the same signal intensity between two images profiles, however, some of the spots exhibited differences. We assessed these differences statistically using the unpaired 
Table 1

The designed primers were used for semi-quantitative RT-PCR

\begin{tabular}{|c|c|c|c|}
\hline Name & Primer & Sequences $\left(5^{\prime} \rightarrow 3^{\prime}\right)$ & Annealing $\left({ }^{\circ} \mathrm{C}\right)$ \\
\hline cycB2 & $\begin{array}{l}\text { Forward } \\
\text { Reverse }\end{array}$ & $\begin{array}{l}\text { TGCCTACAACAGTGCTGAAGTTAGA } \\
\text { CCCCAGCTTTTGATGCTCTT }\end{array}$ & 55 \\
\hline FTH1 & $\begin{array}{l}\text { Forward } \\
\text { Reverse }\end{array}$ & $\begin{array}{l}\text { GGAGCGTGAACATGCTGAGA } \\
\text { CTCCATTGCAGTCAGTCCATTC }\end{array}$ & 56 \\
\hline Gag-Pol & $\begin{array}{l}\text { Forward } \\
\text { Reverse }\end{array}$ & $\begin{array}{l}\text { CCACTGAGATCCGAAAGATCGT } \\
\text { GTGGGCGATGACTGAGTTGA }\end{array}$ & 55 \\
\hline TB4 & $\begin{array}{l}\text { Forward } \\
\text { Reverse }\end{array}$ & $\begin{array}{l}\text { CTCCGCAACCATGTCTGACA } \\
\text { GATTCGCCTGCTTGCTTCTC }\end{array}$ & 58 \\
\hline EEF1A1 & $\begin{array}{l}\text { Forward } \\
\text { Reverse }\end{array}$ & $\begin{array}{l}\text { TGCTGGTGTTGGTGAGTTTGA } \\
\text { GTGGCTCGGTGGAATCCA }\end{array}$ & 60 \\
\hline TXN & $\begin{array}{l}\text { Forward } \\
\text { Reverse }\end{array}$ & $\begin{array}{l}\text { TTGGTGATGTGGTGTTCATTGAA } \\
\text { TTATTGGCCCCAGAGAATTCC }\end{array}$ & 62 \\
\hline ACADL & $\begin{array}{l}\text { Forward } \\
\text { Reverse }\end{array}$ & $\begin{array}{l}\text { TCAAGAGTGGTCGGTGTCGATA } \\
\text { CGGAATGGAGGCTGAATCCT }\end{array}$ & 58 \\
\hline ING4 & $\begin{array}{l}\text { Forward } \\
\text { Reverse }\end{array}$ & $\begin{array}{l}\text { GATATGCCTGTGGACCCCAAT } \\
\text { CAGACCCACACAGGCAAAATG }\end{array}$ & 57 \\
\hline ANXA2 & $\begin{array}{l}\text { Forward } \\
\text { Reverse }\end{array}$ & $\begin{array}{l}\text { GCATATGCCACAGTTAAGGCTTAC } \\
\text { TCATTGCTGCGGTTTGTCA }\end{array}$ & 56 \\
\hline $18 \mathrm{~S}$ rRNA & $\begin{array}{l}\text { Forward } \\
\text { Reverse }\end{array}$ & $\begin{array}{l}\text { CTCCCCTCCCGTTACTTGGA } \\
\text { TTGGTTTTGGTCTGATAAATGCA }\end{array}$ & 58 \\
\hline
\end{tabular}

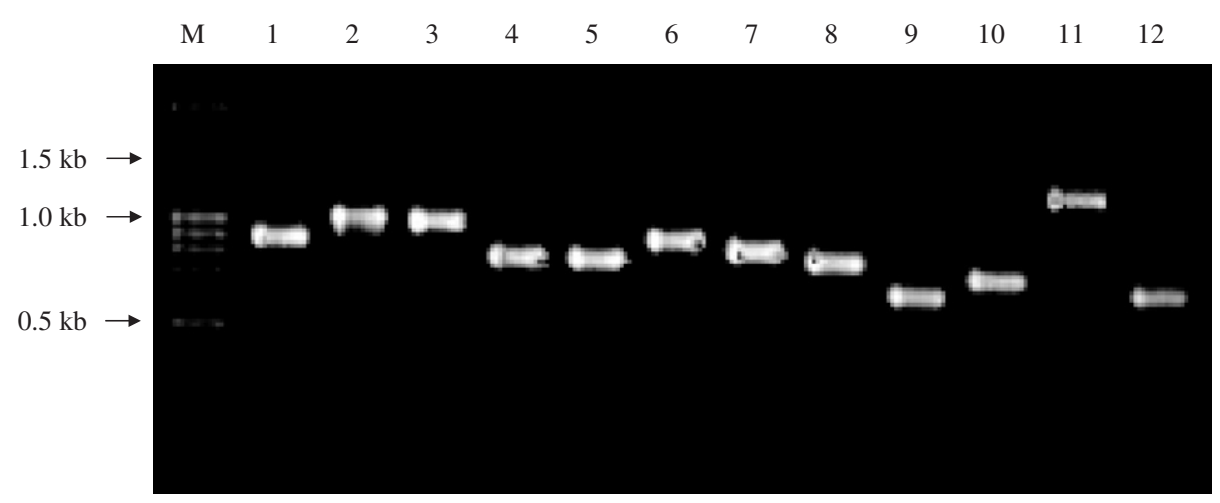

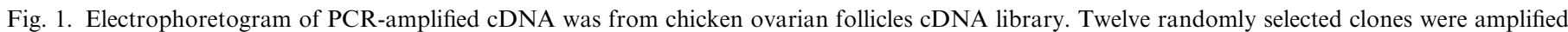
with SP6 and T7 primers and the inserts size were showed in range from 0.5 to $1.5 \mathrm{~kb}$. M: Bio- 100 ladder marker.

t-test in Avadis version 3.3 software (Strand Life Sciences, Redwood City, CA, USA). This analysis revealed that 257 $(6.7 \%)$ transcripts were differentially expressed $(P<0.05)$ (Fig. 2a). Further assessment established that $48(1.2 \%)$ transcripts were differentially expressed by a factor of greater than 1.75-fold or less than -1.75-fold in log intensity (Fig. 2b). Twenty-seven of these 48 transcripts were enriched in ovarian follicles from the B chicken strain, while 21 transcripts were highly expressed in the L2 strain.

\subsection{Identification of differentially expressed transcripts}

The 48 differentially expressed transcripts were subjected to DNA sequence analysis using the GenBank BLASTN program. This showed 20 sequences were non-redundant transcripts (Table 2). Eight transcripts were highly expressed in chicken strain B and were identified as cyclin B2 (cycB2), Gag-Pol polyprotein, elongation factor $1 \propto 1$ (EEF1A1), hemopexin (HPX), ferritin heavy polypeptide 1 (FTH1), nucleoside diphosphate kinase (CNDPK), thymosin $\beta 4$ (TB4) and small EDRK-rich factor 1 (Serf1). Twelve transcripts were enriched in the L2 strain material and these consisted of aquaporin 1 (Aqp1), annexin II (ANXA2), immunoglobulin heavy- and light-chain, thioredoxin (TXN), $\alpha$-tropomyosin (TPM1), inhibitor of growth family member 4 (ING4), acetyl-coenzyme A dehydrogenase, long chain (ACADL), and ribosomal proteins L14, L18a, L22 and L23. 


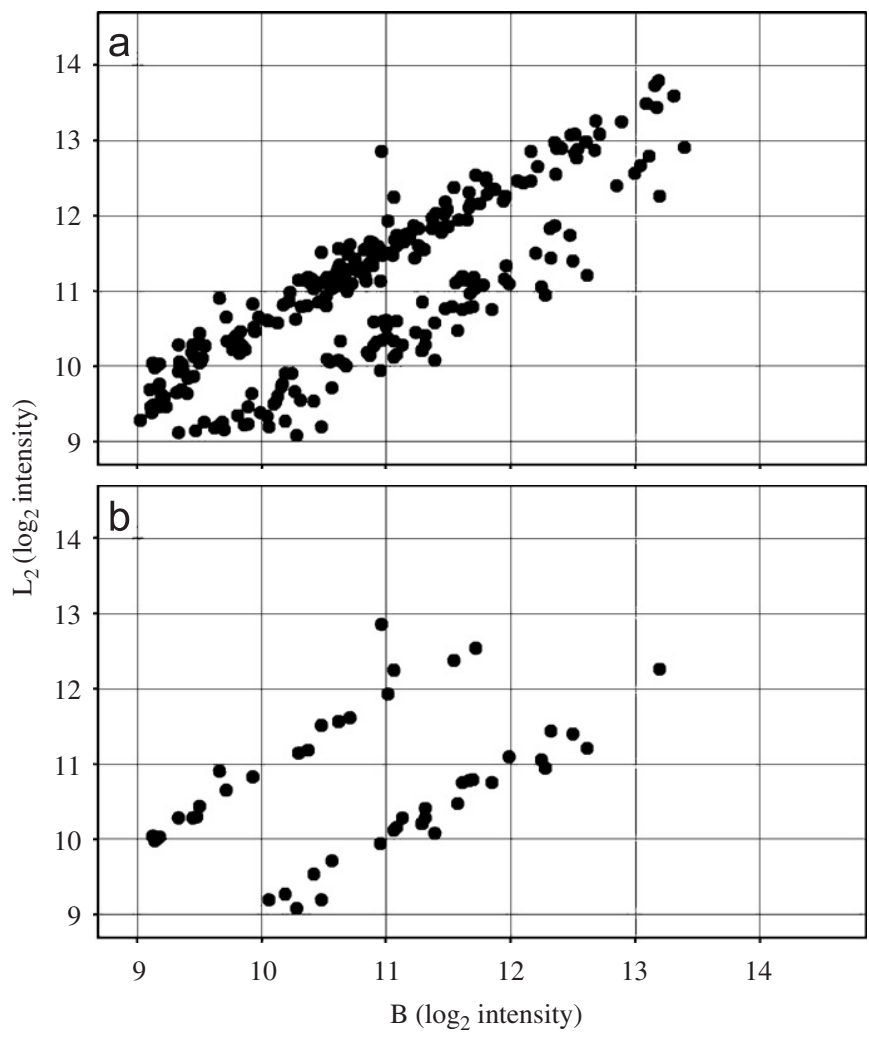

Fig. 2. Scatter plots of logarithmic fluorescent intensity from normalized cDNA microarrays. (a) There were 257 differentially expressed transcripts $(P<0.05)$ screened in both ovarian follicles of Taiwan country chickens strains by the Avadis software analyzed. (b) Filtered 48 from 257 differentially expressed transcripts using the cutoff method by the $\log _{2}$ intensities greater than 1.75 -fold or less than -1.75 -fold.

\subsection{Semi-quantitative RT-PCR validation}

In order to confirm that the sequences we had identified by sequencing analysis were identical to the transcripts initially identified from our microarrays, we went back to our preparations of ovarian RNA and used semi-quantitative RT-PCR to confirm that we could amplify the differentially expressed transcripts in this material. Nine transcripts including cyclin B2 (cycB2), Gag-Pol polyprotein, ferritin heavy peptide 1 (FTH1), thymosin $\beta 4$ (TB4), elongation factor $1 \alpha 1$ (EEF1A1), thioredoxin (TXN), annexin II (ANXA2), inhibitor of growth family member 4 (ING4) and acetyl-coA dehydrogenase long chain (ACADL) transcripts were selected for analysis. Amplification of $18 \mathrm{~S}$ rRNA was used as a positive control in all the semi-quantitative RT-PCR experiments. We found the semi-quantitative RT-PCR results of all the nine selected transcripts matched the expression profiles established with the cDNA microarray data (Fig. 3). These results confirmed that our approach using cDNA microarrays was a reliable and valuable way to identify differentially expressed transcripts in chicken ovarian follicles.

\section{Discussion}

The development of cDNA microarray technology has allowed large scale variations in the patterns of mRNA expression to be observed in a single experiment [7]. The general approach for cDNA microarray analysis has the following stages: (1) cDNA library construction, (2) EST sequence analysis, (3) sequence annotation with GenBank

Table 2

Differentially expressed transcripts in chicken ovaries from B and L2 strains

\begin{tabular}{|c|c|c|c|c|c|}
\hline Accession no. & Description & Symbol & $\log _{2}$ ratio $(\mathrm{B} / \mathrm{L} 2)$ & Organism & Category \\
\hline \multicolumn{6}{|c|}{ Enrich in $B$ strain } \\
\hline EC912003 & Cyclin B2 & сусB2 & 2.01 & Gallus gallus & Cell cycle \\
\hline EC912005 & Elongation factor $1 \alpha 1$ & EEF1A1 & 2.00 & Gallus gallus & Protein biosynthesis \\
\hline EC912006 & Hemopexin & HPX & 1.80 & Gallus gallus & Transport \\
\hline EC912007 & Ferritin heavy polypeptide 1 & FTH1 & 1.98 & Gallus gallus & Immune response \\
\hline EC912010 & Small EDRK-rich factor 1 & SERF1 & 2.04 & Mus musculus & Nervous system development \\
\hline \multicolumn{6}{|c|}{ Enrich in L2 strain } \\
\hline EC912011 & Aquaporin 1 & AQP1 & -1.81 & Bos taurus & Water transport \\
\hline EC912012 & Annexin II & ANXA2 & -1.89 & Gallus gallus & Angiognenesis \\
\hline EC912013 & IgG heavy chain (HC36) D-J-C & & -1.92 & Gallus gallus & Immune response \\
\hline EC912014 & Thioredoxin & TXN & -1.88 & Gallus gallus & Cell motility/cell proliferation \\
\hline EC912019 & Ribosomal protein L22 & RPL22 & -1.79 & Homo sapiens & Structural constituent of ribosome \\
\hline EC912020 & Acetyl-CoA dehydrogenase, long chain & ACADL & -1.91 & Mus musculus & Fatty acid metabolism \\
\hline EC912021 & Ribosomal protein L23 & RPL23 & -1.75 & Rattus norvegicus & Structural constituent of ribosome \\
\hline EC912022 & Ribosomal protein L18a & RPL18a & -2.37 & Rattus norvegicus & Structural constituent of ribosome \\
\hline
\end{tabular}




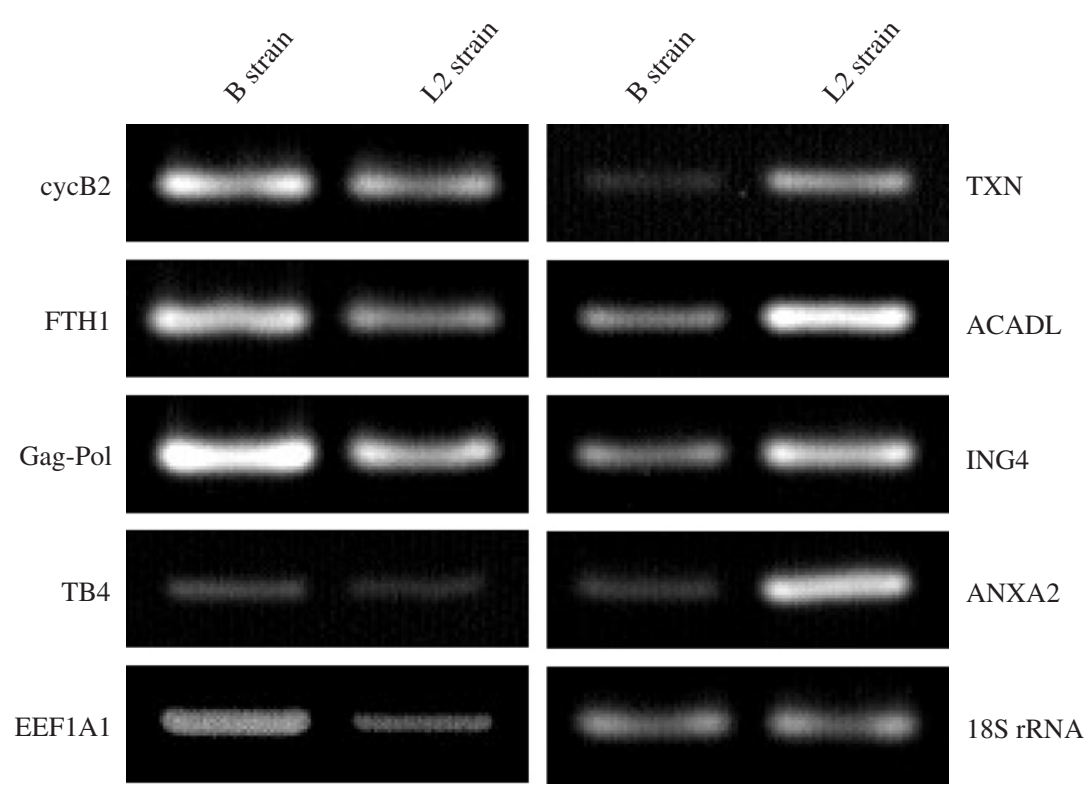

Fig. 3. Semi-quantitative RT-PCR analysis of mRNA expression was based on cDNA microarrays. The transcripts levels of cyclin B2 (cycB2), ferritin heavy polypeptide 1 (FTH1), Gag-Pol polyprotein, thymosin $\beta 4$ (TB4) and elongation factor $1 \alpha 1$ (EEF1A1) were higher expressed in ovarian follicles from B strain, whereas thioredoxin (TXN), actyl-CoA dehydrogenase long chain (ACADL), inhibitor of growth family member 4 (ING4) and annexin II (ANXA2) were expressed in L2 strain at higher levels. These semi-quantitative RT-PCR results that showed the transcripts expression changes were corresponded to cDNA microarrays. The $18 \mathrm{~S}$ rRNA was used for internal control.

and (4) the non-redundant transcripts amplified by PCR before spotting. In this study, we followed this general protocol and constructed chicken ovarian follicle cDNA libraries derived from two strains of Taiwan country chickens using tissue isolated from ovarian follicles and connective tissue from hens sacrificed at egg-laying stages. Due to the high cost of commercial DNA chips and sequence analysis, we used a previously published and successful method of "in house" cDNA microarray analysis in the current experiment [12,22]. The majority of the amplified cDNA inserts that we isolated from the 3840 randomly selected clones and used for our experiment were larger than $500 \mathrm{bp}$ (Fig. 1). This finding supported our hypothesis that these inserts were significant and merited further analysis. Furthermore, quality control measures showed that our "in house" cDNA microarray data was highly reproducible (data not shown). Using these methods we characterized differential expression profiles of RNA transcripts detected in ovarian follicles from two strains of chicken which had either high (L2) or low (B) rates of egg production. The expression profiles between the B and L2 strains showed many general similarities because the birds share the same genetic background [2]. This meant that the number of differentially expressed clones was limited, but despite this a number of novel and interesting transcripts were found.

Differentially expressed sequences were aligned using the BLAST programme and annotated by Gene Ontology (http://www.geneontology.org/). We identified eigth transcripts enriched in strain B ovarian follicles, which has the lower rate of egg production, and 12 transcripts were differentially expressed in the L2 strain (Table 2). Some of the sequences we isolated in this experiment have been previously described during oogenesis and folliculogenesis, although others have not (see Table 2). After identifying differentially expressed sequences in strain B we went on to confirm our findings with semi-quantitative RT-PCR (Fig. 3). Interestingly, we detected enriched expression of two mRNAs, cyclin B2 (cycB2) and elongation factor $1 \propto 1$ (EEF1A1), in the B strain that have been previously described in oogenesis. $\mathrm{CycB} 2$ is present in immature oocytes during porcine oogenesis [25], and EEF1A1 mRNA has been described in previtellogenic (immature) oocytes during oogenesis and embryo development [26]. This suggests that there may be more immature oocytes in the less productive $B$ strain than in the L2 strain associated with higher rates of egg production. We also found enriched expression levels of thymosin $\beta 4$ (TB4), plasma levels of which have been proposed to influence ovarian activity, although the underlying mechanism is not fully understood. Levels of TB4 increase in ovaries of domestic hens during moulting [27], and since we detected it at increased levels in the B strain, this may indicate that TB4 may be a potentially important marker associated with the lower rates of egg production in the B chicken strain. We also found that ferritin transcripts were enriched in the B strain. Ferritin is a female-specific gene product highly expressed in the vitellarium, which decreases post-ovulation [28]. Our finding suggests that the higher expression levels of ferritin present in the B strain may delay ovulation compared to the L2 strain, and so may be also related to the different rates of egg production in the two chicken strains. 
We isolated a number of transcripts that were enriched in the L2 chicken strain (see Table 2), and were able to confirm these findings with semi-quantitative RT-PCR (Fig. 3). The function of many of these transcripts are consistent with a role in efficient egg production (Table 2). Thioredoxin (TXN), is highly expressed in the ovary of L2 chickens and may play an important part in antioxidative defense [29]. During normal folliculogenesis fluid collects in the ovarian follicle via a transcellular transport mechanism mediated through aquaporin [30]. Aquaporin 1 (AQP1) is a membrane protein that controls the permeability of endothelial and epithelial barriers by facilitating water movement across cell membranes [31]. Tropomyosin (TPM1) and troponin are major actinlinked regulators of ovarian muscle contraction in the nematode Caenorhabdities elegans and it has been suggested that their action enhances release of mature oocytes [32]. High levels of immunoglobulin ( $\operatorname{IgG})$ have been described in ovarian tissue isolated from laying hens which have high egg production rates [33], and it has been proposed that efficient ovarian function requires an effective immune system. We found that long chain acetyl-coA dehydrogenase (ACADL), involved in the beta-oxidation of fatty acids, was highly expressed in L2 ovarian follicles which suggested that fatty acids may be utilized as an energy source to ensure rapid folliculogenesis [34]. Annexin II (AXNA2) has been proposed to play an important regulatory role during oocyte maturation in mammals [35], and may carry out a similar function during oocyte development in the L2 strain of chickens.

We also found that two transcripts, Gag-Pol polyprotein and inhibitor of growth family member 4 (ING4), which share homology with the Homo sapiens sequences, were differentially expressed in the B and L2 strains, respectively (Table 2, Fig. 3). This is the first time that the expression of these transcripts has been described in chicken ovarian follicles. The Gag-Pol polyprotein is required for proteolytic processing of the human immunodeficiency virus type 1 (HIV-1) [36] and ING4 is a candidate tumor suppressor protein [37]. Our findings suggest that Gag-Pol polyprotein and ING4 are novel molecular regulators related to egg production in the ovarian follicles of Taiwan country chickens.

The present study is the first application of our in-house cDNA microarrays to evaluate differences in the global transcriptome between ovarian follicles isolated from Taiwan country chickens that have either low or high rates of egg production. We have demonstrated that our techniques represent an effective and reliable method to characterize and profile gene transcripts expressed during the physiological process of egg production. We have been able to identify gene transcripts that have been previously reported in ovarian function, as well as isolating new candidates. These transcripts may be suitable as early selection markers in breeding programs for Taiwan country chickens.

\section{References}

[1] Fairfull RW, Gowe RS. Genetics of egg production in chickens. In: Crawford RD, editor. Poultry breeding and genetics. Amsterdam: Elsevier Science Publishers; 1990. p. 705-59.

[2] Lee YP. Development and improvement of local chicken in Taiwan. In: Proceedings of the 5th AAAP Animal Science Congress; 1990. p. $975-9$.

[3] Su H, Silversides FG, Villeneuve P. Ovarian follicular growth and maturity and follicular production of progesterone and oestradiol in response to porcine luteinising hormone and porcine follicle stimulating hormone in albino $\left(S^{*} A S\right)$ hens in vivo and in vitro. $\mathrm{Br}$ Poult Sci 1999;40:545-51.

[4] Eppig JJ, Wigglesworth K, Pendola FL. The mammalian oocyte orchestrates the rate of ovarian follicular development. Proc Natl Acad Sci USA 2002;99:2890-4.

[5] Hocking PM, Bernard R, Robertson GW. Effects of low dietary protein and different allocations of food during rearing and restricted feeding after peak rate of lay on egg production, fertility and hatchability in female broiler breeders. Br Poult Sci 2002;43:94-103.

[6] Ciacciariello M, Gous RM. A comparison of the effects of feeding treatments and lighting on age at first egg and subsequent laying performance and carcase composition of broiler breeding hens. $\mathrm{Br}$ Poult Sci 2005;46:246-54.

[7] Schena M, Shalon D, Davi RW, Brown PO. Quantitative monitoring of gene expression patterns with a complementary DNA microarray. Science 1995;270:467-70.

[8] Duggan DJ, Bittnet M, Chen Y, Meltzer P, Trent JM. Expression profiling using cDNA microarrays. Nat Genet 1999;21:10-4.

[9] Leo CP, Pisarska MD, Hsueh AJW. DNA array analysis of changes in preovulatory gene expression in the rat ovary. Biol Reprod 2001;65:269-76.

[10] Lee BC, Cha K, Avraham S, Avraham HK. Microarray analysis of differentially expressed genes associated with human ovarian cancer. Int J Oncol 2004;24:847-51.

[11] Ponsuksili S, Wimmers K, Adjaye J, Schellander K. A source for expression profiling in single preimplantation bovine embryos. Theriogenology 2002;57:1611-24.

[12] Yao J, Ren X, Ireland JJ, Coussens PM, Smith TPL, Smith GW. Generation of a bovine oocyte cDNA library and microarray: resources for identification of genes important for follicular development and early embryogenesis. Physiol Genomics 2004;19:84-92.

[13] Caetano AR, Johnson RK, Ford JJ, Pomp D. Microarray profiling for differential gene expression in ovaries and ovarian follicles of pigs selected for increased ovulation rate. Genetics 2004;168:1527-37.

[14] Lee SH, Zhao SH, Recknor JC, Nettleton D, Orley S, Kang SK, et al. Transcriptional profiling using a novel cDNA array identifies differential gene expression during porcine embryo elongation. Mol Reprod Dev 2005;71:129-39.

[15] Hatey F, Tosser-Klopp G, Clonscard-Martinato C, Mulsant P, Gasser F. Expressed sequence tags for genes: a review. Genet Sel Evol 1998:30:521-41.

[16] Tirunagaru VG, Sofer L, Cui J, Burnside J. An expressed sequence tag database of $T$-cell enriched activated chicken splenoctyes: sequence analysis of 5251 clones. Genomics 2000;66:144-51.

[17] Abdrakhmanov I, Lodygin D, Geroth P, Arakawa H, Law A, Plachy $\mathrm{J}$, et al. A large database of chicken bursal ESTs as a resource for the analysis of vertebrate gene function. Genome Res 2000;10:2062-9.

[18] Smith EJ, Shi L, Drummond P, Rodriguez L, Hamilton R, Ramlal S, et al. Expressed sequence tags for chicken genome from a normalized 10-day-old white leghorn whole embryo cDNA library: 1. DNA sequence characterization and linkage analysis. J Hered 2001;92:1-8.

[19] Smith EJ, Shi L, Prevest L, Drummond P, Ramlal S, Smith G, et al. Expressed sequence tags for chicken genome from a normalized 10-day-old white leghorn whole embryo cDNA library: 2. Comparative DNA sequence analysis of guinea fowl, quail and turkey genomes. Poult Sci 2001;80:1263-72. 
[20] Carre W, Diot C, Fillon V, Crooijmans RP, Lagarrigus S, Morrisson $\mathrm{M}$, et al. Development of 112 unique expressed sequence tags from chicken liver using an arbitrarily primed reverse transcriptase polymerase chain reaction and single strand conformation gel purification method. Anim Genet 2001;32:289-97.

[21] Cogburn LA, Wang X, Carre W, Rejto L, Porter TE, Aggrey SE, et al. Systems-wide chicken DNA microarrays, gene expression profiling, and discovery of functional genes. Poult Sci 2003;82:939-51.

[22] van Hemert S, Ebbelaar BH, Smits MA, Rebel JMJ. Generation of EST and microarray resources for functional genomic studies on chicken intestinal health. Anim Biotechnol 2003;14:133-43.

[23] Ewing B, Hillier L, Wendl MC, Green P. Base-calling of automated sequencer traces using phred. I. Accuracy assessment. Genome Res 1998;8:175-85.

[24] Serazin-Leroy V, Denis-Henriot D, Morot M, de Mazancourt P, Giudicelli Y. Semi-quantitative RT-PCR for comparison of mRNAs in cells with different amounts of housekeeping gene transcripts. Mol Cell Probes 1998;12:283-91.

[25] Kuroda T, Naito K, Sugiura K, Yamashita M, Takakura I, Tojo H. Analysis of the roles of cyclin B1 and cyclin B2 in porcine oocyte maturation by inhibiting synthesis with antisense RNA injection. Biol Reprod 2004;70:154-9.

[26] Poting A, Danker K, Hartmann L, Koster M, Wedlich D, Knochel W. Two different mRNAs coding for identical elongation factor 1 alpha (EF-1 alpha) polypeptides in Xenopus laevis embryos. Differentiation 1990;44:103-10.

[27] Dickerman RW, Wise TH, Bahr JM. Effect of ovarian regression and molt on plasma concentrations of thymosin beta 4 in domestic hens (Gallus domesticus). Domest Anim Endocrinol 1992;9:297-304.

[28] Aegerter S, Jalabert B, Bobe J. Large scale real-time PCR analysis of mRNA abundance in rainbow trout eggs in relationship with egg quality and postovulatory ageing. Mol Reprod Dev 2005;72:377-85.
[29] Zieker D, Fehrenbach E, Dietzsch J, Fliegner J, Waidmann M, Nieselt K, et al. cDNA microarray analysis reveals novel candidate genes expressed in human peripheral blood following exhaustive exercise. Physiol Genomics 2005;23:287-94.

[30] McConnell NA, Yunus RS, Gross SA, Bost KL, Clemens MG, Hughes Jr FM. Water permeability of an ovarian antral follicle is predominantly transcellular and mediated by aquaporin. Endocrinology 2002;143:2905-12.

[31] Mobasheri A, Airley R, Hewitt SM, Marples D. Heterogeneous expression of the aquaporin 1 (AQP1) water channel in tumor of the prostate, breast, ovary, colon and lung: a study using high density multiple human tumor tissue microarrays. Int J Oncol 2005;26: $1149-58$

[32] Ono K, Ono S. Tropomyosin and torponin are required for ovarian contraction in the Caenorhabditis elegans reproductive system. Mol Biol Cell 2004;15:2782-93.

[33] Barua A, Yoshimura Y. Ovarian autoimmunity in relation to egg production in laying hens. Reproduction 2001;121:117-22.

[34] Seol HS, Sato K, Murakami H, Toyomizu M, Akiba Y. Changes in gene expression involved in energy utilization during chicken follicle development. Anim Reprod Sci 2006;95:283-94.

[35] Cui XS, Song H, Kim NH. Identification of metaphase II-specific gene transcripts in porcine oocytes and their expression in early stage embryos. Reprod Fertil Dev 2005;17:625-31.

[36] Tachedjian G, Moore KL, Goff SP, Sluis-Cremer N. Efavirenz enhances the proteolytic processing of an HIV-1 pol polyprotein precursor and reverse transcriptase homodimer formation. FEBS Letters 2005;579:379-84.

[37] Garkavtsev I, Kozin SV, Chernova O, Xu L, Winkler F, Brown E, et al. The candidate tumor suppressor protein ING4 regulates brain tumor growth and angiogenesis. Nature 2004;428: $328-32$. 